\title{
Experimental Research into the Influence of Different Athletic Loads on the Activities of Serum Enzyme of Non-Exercising Rats
}

\author{
Yanjie Jiang \\ Institute of Physical Education, Changchun Normal University, Changchun Jilin Province ,China \\ jiangyanjie@sina.com
}

\begin{abstract}
Keywords:Overload exercises; Immunoglobulin G; Creatine Kinase; Lactate Dehydrogenass; Glutamic-pyruvic transaminase; Glutamic-pyruvic transaminase
\end{abstract}

\begin{abstract}
Purpose: to discuss the biologic and chemical mechanism of the influence on the organisms' health exerted by different athletic loads. Method: experimental method is adopted to observe the influence of different athletic loads for six weeks continuously on such indices as IgG, CK, LDH, GPT-ALT and so on in the rats' blood. Results: The IgG in the rats' serum of 15 normal exercise rats in this test significantly rises with significant difference compared with the control group $(\mathrm{P}<0.10)$; CK, LDH and GPT-ALT in the serum of the 15 rats that have done overload exercises significantly rise, but IgG falls significantly with significant difference compared with the $\mathrm{C}$ (control group) or $\mathrm{NE}$ (normal exercise) $(\mathrm{P}<0.05$ and $\mathrm{P}<0.01)$. Conclusion: normal exercises done by non-exercising rats may help to improve their immunity, however, centralized overload exercises may weaken their immunity and injure the skeletal muscle cells and liver cells.
\end{abstract}

\section{Introduction}

By observing the influence of different athletic loads of rats that have done exercises continuously for six weeks on such indices as CK, LDH, GPT-ATL, IgG, the influence of normal exercises on the organisms' immunity and the mechanism of the damage of centralized overload exercises to the organisms' health are discussed, thus helping people to avoid ineffective exercises and damage arising from exercises, and providing theoretical basis for the right amount of scientific fitness exercises.

\section{Materials and Methods}

Experimental Animals and Their Feeding.52 6-8 weeks' male wistar rats of 232 \pm 12.9 grams are selected to feed in different cages in ordinary ways and are fed freely.

Experimental Apparatuses. ZH-PT animal treadmill manufactured by Huaibei Zhenghua Biological Instrument and Apparatus Co., Ltd.

Preparing for Animal Models that do Normal and Overload Exercises and Grouping the Animals. After the rats are bought, the rats are fed adaptively for one week. They are classified into three groups. (1) Control group are fed in an ordinary way without interruption. (2) the normal exercise group (NE) do exercises on the moderate treadmill at the speed of $15 \mathrm{~m} \cdot \mathrm{min}-1$. They do exercises for 10 minutes for the first time, and then the duration increases by $5 \mathrm{~min}$ day by day until the second weekend when they do exercises for 60 min every day (have a rest for 10 min after 30 min exercises, and then do exercise for another $30 \mathrm{~min}$ ), and then remain this amount of exercises until the expiration of six weeks. (3) The overdue exercise group does exercises twice every week at the speed of $20 \mathrm{~m} \cdot \mathrm{min}-1$ until exhaustion; after they do exercises for $20 \mathrm{~min}$ for the first time, the duration increases day by day, and on the second weekend (namely, Saturday and Sunday) they work for more than 200 min every time; after 100 min exercises, the rats have a rest for $10 \mathrm{~min}$ and then do exercises for another $100 \mathrm{~min}$. The rats will be considered exhausted when sound, light or brush is used for stimulating their tails, and some rats fail to do exercises for $200 \mathrm{~min}$, although stimulated, they fail to maintain the exercise strength specified or fail to do exercises at all, and their exercises are terminated in advance, the duration is recorded and for the rest time, they are fed in different cages. Before the 
test is done on the overload exercise group and the normal exercise group, they have two days to get familiar with the treadmill exercises.

Execution and Taking Materials.After the exercise groups do exercise for 6 weeks, they are executed 24 hours after doing exercises, and Pelltobarbitalum Natricum of 3\% is injected into the abdominal cavity (I mL per $\mathrm{kg}$ of weight), and heart blood is sampled from the three groups of rats, and the quantity of blood sampled from each rat is at least $5 \mathrm{ml}$, including $2 \mathrm{ml}$ of anticoagulant blood that is used for measuring $\mathrm{HGB}, \mathrm{HCT}, \mathrm{WBC}$ and $\mathrm{RBC}$, and $3 \mathrm{ml}$ of self-coagulation blood used for measuring BUN, IgG, CK, LDH, GPT-ATL.

Testing Method of Biochemical Indices in the Blood and Serum.HGB, HCT, WBC, RBC: measured by using the JT-IR full-automatic blood- cell counter supplied by Backman (USA). BUN, IgG, LDH and GPT-ALT: measured by CX-9 full-automatic biochemistry analyzer from Backman (USA).CK is measured by using the radioimmunoassay method; the immunoassay kits are supplied by the isotope room of China Institute of Atomic Energy and Jiuding Diagnostic. All of the indices above are tested by the Department of Clinical Laboratories of No. 1 Clinical Hospital of Jilin University.

Statistic Treatment. Data statistics and analysis are completed by using the statistic analysis software of SPSS19.0, and one-way ANOVA is used for inter-group comparison.

\section{Experimental Results}

Change in WBC, RBC and HGB in the Blood of the Three Groups of Rats.WBC and RBC of the 15 normal exercise rats in this test rise with significant difference compared with that of the control group $(\mathrm{P}<0.01)$. For the 15 overload exercise rats, WBC rises and HGB falls with significant difference compared with those of the control group $(\mathrm{P}<0.01)$, the decrement of HGB is bigger also with significant difference compared with that of NE group $(\mathrm{P}<0.01)$. However, the change in RBC is small without statistic significance (Table 1).

Change in CK, LDH, GPT-ALT, BUN and lgG in the blood of the three groups of rats.

Table1 Change in WBC, RBC, HGB and HCT in the Blood of the Three Groups of Rats $(\mathrm{n}=15, \bar{x} \pm \mathrm{s})$

\begin{tabular}{|l|l|l|l|l|}
\hline Group & \multicolumn{1}{|c|}{$\begin{array}{c}\text { WBC } \\
(109 \cdot \mathrm{L}-1)\end{array}$} & $\begin{array}{c}\text { RBC } \\
(1012 \cdot \mathrm{L}-1)\end{array}$ & $\begin{array}{c}\text { HGB } \\
(\rho \mathrm{B} / \mathrm{g} \cdot \mathrm{L}-1)\end{array}$ & HCT \\
\hline C & $6.94 \pm 0.72$ & $6.25 \pm 0.52$ & $115.95 \pm 1.28$ & $55.91 \pm 1.45$ \\
\hline OE & $11.14 \pm 1.72 * *$ & $7.32 \pm 0.55$ & $105.05 \pm 5.01 * * \# \#$ & $52.26 \pm 1.27$ \\
\hline NE & $9.94 \pm 0.69 * *$ & $9.31 \pm 1.51^{* *}$ & $127.76 \pm 10.22$ & $65.02 \pm 3.68$ \\
\hline
\end{tabular}

${ }^{* *} \mathrm{P}<0.01$ compared with the control group; ${ }^{\#} \mathrm{P}<0.05{ }^{\# \#} \mathrm{p}<0.01$ compared with the normal exercise group

Table2 Change in CK, IgG,LDH-L,GPT-ALT and BUN in the Blood of the Three Groups of $\operatorname{Rats}\left(\mathrm{n}=15, \bar{x}_{ \pm \mathrm{s}}\right)$

\begin{tabular}{|l|l|l|l|l|l|}
\hline Group & \multicolumn{1}{|c|}{$\begin{array}{c}\text { CK } \\
(\lambda \mathrm{B} / \mathrm{U} \cdot \mathrm{L}-1)\end{array}$} & $\begin{array}{c}\text { IgG } \\
(\rho \mathrm{B} / \mathrm{g} \cdot \mathrm{L}-1)\end{array}$ & $\begin{array}{c}\text { LDH-L } \\
(\lambda \mathrm{B} / \mathrm{U} \cdot \mathrm{L}-1)\end{array}$ & $\begin{array}{c}\text { GPT-ALT } \\
(\lambda \mathrm{B} / \mathrm{U} \cdot \mathrm{L}-1)\end{array}$ & $\begin{array}{c}\text { BUN } \\
(\mathrm{cB} / \mathrm{mmol} \cdot \mathrm{L}-1)\end{array}$ \\
\hline $\mathrm{C}$ & $216.23 \pm 62.88$ & $2.96 \pm 0.25$ & $63.93 \pm 3.82$ & $26.32 \pm 1.29$ & $5.39 \pm 0.86$ \\
\hline OE & $350.45 \pm 69.19 * * \# \#$ & $2.92 \pm 0.21^{\# \#}$ & $68.13 \pm 5.16^{*}$ & $44.65 \pm 1.81^{* * * \#}$ & $7.06 \pm 0.52 * * \# \#$ \\
\hline NE & $227.56 \pm 56.02$ & $4.71 \pm 1.13^{* *}$ & $58.56 \pm 6.27$ & $29.29 \pm 5.56^{* *}$ & $5.51 \pm 0.65$ \\
\hline${ }^{*} \mathrm{P}<0.05^{* *} \mathrm{P}<0.01$, compared with the control group; ${ }^{\#} \mathrm{P}<0.05^{\# \#} \mathrm{p}<0.01$ compared with the normal exercise group
\end{tabular}

IgG and GPT-ALT of the 15 normal exercise rats in this test rise with significant difference compared with the control group $(\mathrm{P}<0.01)$; $\mathrm{CK}$ and $\mathrm{LDH}$ of the 15 overload exercise rats rise with 
significant difference compared with the normal exercise group and the control group $(\mathrm{P}<0.05)$; BUN and GPT-ALT of the overload exercise group are much higher than those of the normal exercise group and the control group with significant difference $(\mathrm{P}<0.10)$ and $\mathrm{IgG}$ of the overload exercise group is much lower than that of the normal exercise group $(\mathrm{P}<0.01)$ (Table 2$)$.

\section{Discussion}

Since physical fitness programmes involving all our people started, more and more people become aware of the importance of sports to health, even when the work and life pace go fast, they also reserve some time for doing exercises. However, exercises shall be done under the scientific guidance, and it is not acceptable for some people to do overload exercises on the weekend or in holidays to make up for the exercises that should have been done but have not been done at other time. In recent years, there are a lot of researches on the overload exercises done by sportsmen, but there are few researches on the overload exercises done by common people. As the tide of the physical fitness programmes involving all our people rises, this issue attracts the attention of more and more people [1]. According to the conclusions of this paper, for common people, right amount of exercises may help to improve the organism's immunity, however, overload exercise will cause the immunity to fall, and damage to muscle cells and liver cells.

There have been different reports on the influence of exercises on IgG by scholars both inside and outside China. As early as the 20th century, there had been reports that physical exercises have some influence on the organisms' immunities, and right amount of exercises may reduce the risks and severity of infection [2]. It is found according to the test on normal students' and Tai Ji Quan sportsmen's immune indices, the content of IgG, IgA and IgM in the serum of the experimental group that does Tai Ji Quan for a long term is significantly higher than that of the control group, whether before or after doing the exercises. According to the research results, right amount of long term exercises may enhance the organisms' immunity, which can be seen from the experiment results on the normal exercise group. Long-term intense exercises exert great negative influence on the immunity. According to the researches that study the influence of different amounts of exercises on the animals' immunity indices such as Pu Junzong's research: after five weeks' swimming exercises, the antibody response caused by the small rats'antigen is inhibited [4]. The IgG measured of the rats that have done overload exercises is low with significant difference with that of the normal exercises, which means that overload exercises may reduce the organisms' immunity.

CK widely exists in human bodies' skeletal muscles, heart muscles, brains and smooth muscles, and the skeletal muscles contain the most CK; most of CK in the serum comes from the skeletal muscles [3] [4]. In normal cases, the muscle cells are integral with normal functions so that little CK expels through the cell membrane. A lot of exercises will improve the activity of CK. As the number of CKs in the muscle cells is significantly different from that in the blood, the change in the CK activity in the serum may be taken as the important and sensitive biochemical indices for assessing the stimulus that muscles receive and knowing the tiny damage to the muscles as well as its adaptation and recovery [5]. There are also other researches showing that after submaximal exercise done by sportsmen and healthy non-sportsmen, the level of CK in serum greatly depends on the duration of the exercises [6]. It is found according to some scholars' researches on the CK in the Charles River rats' plasma and the enzymes that perform the same biochemical functions after they swim[7], the activity of $\mathrm{CK}$ in the serum after doing exercises significantly rises and this level of CK will remain for some time and then will change in the single peak way. As a result, the change in $\mathrm{CK}$ and the enzymes that perform the same biochemical functions after rats or human beings do exercises is associated with the exercise load, however, the sport load is associated with the damage to the skeletal muscles. The CK in the serum of the overload exercises group is higher than those of the normal exercise group $(\mathrm{P}<0.01)$, which means that this kind of overload exercises causes damage to the membranes of the muscle cells. 
LDH is an enzyme for catalyzing the mutual conversion between the lactic acid and the pyruvate. The rise of the activity of LDH in the serum after doing exercises is closely associated with the load of exercises [8]. After marathon or long time walking, the activity of LDH significantly rises with its peak to be observed immediately after the exercises until the next day, and it recovers slowly, which may take several days in some cases. Bi Qiuyun [9] etc has trained the rats by increasing the load gradually. After 5 weeks' exercises with load increased gradually, the activity of LDH, ATL and AST of the rats rises. The LDH and the BUN measured 24 hours after the rats do overload exercises are still very high, which means that the exercise load of the overload exercise group is very large.

GPT-ALT is also called Alanine aminotransferase, and may reversibly catalyze the reaction between the alanine and the ketoglutaric acid, which has the function of linking protein metabolism and carbohydrate metabolism. The conclusions on the influence of exercises on the activities of serum GPT-ALT are not always consistent. However, most researchers think that the exercise load, when increased to a certain extent, may cause the activity of GPT-ALT in the serum to rise during and after the exercises [9]. In addition, the activity of GPT-ALT in the serum changes synchronously with the exercise fatigue, and is also an index for assessing the damage to the liver cells. According to Wang Pei's and Wang Guoqing's [10] researches on the influence of overload exercises on the rats' liver functions and the related biochemical indices such as BUN, LDH, WBC and so on abovementioned, it can be seen that the bodies of the rats that have done overload exercises are tired and their liver cells are damaged.

According to the experiments on human bodies, when sportsmen do heavy load exercises for a long term or do overload exercises, their hemoglobin falls[11]. Ye Jianfei and others have found after taking 3 months' SD male rats to do normal exercises and overdue exercises that the hemoglobin of the normal exercise group is a little higher than that of the quiet control group, and that of the overload exercise group is significantly lower than that of the quiet control group and the normal exercise group [12]. We can often find from the test results that the decrement of HGB of the overload exercise group is significant, and its HGB is lower than that of the normal exercise group, and even lower than that of the quiet control group. To sum up, right amount of exercises may help to increase $\operatorname{IgG}$ in the rats' serum, and their immunities rise, however, centralized overload exercises done twice every week exert negative influence on some biochemical indices, reduce the content of hemoglobin and the immunity, damage the liver cells. It is suggested that common people shall do right amount of exercises in a scientific way, and shall pick up the habit of doing exercises, insist on doing right amount of exercises regularly all their lives, and avoid overload exercises.

\section{References}

[1] Yu Xuehao. Influence of Tai Ji Quan on the Human Bodies' Immunoglobulin Level. Journal of Lingling University, 3 (2009) 128-130

[2] Jannie W,et al. Radioimmunoassay for urinary albumin. Clin Chem, 24(1978)1464.

[3] Paller M, et al. Oxygen free radicals is ischemic acute renal failure in the rat. J Clin Invest,10 (1984)1156.

[4] Yoshihiko Oy anagui. The utilization of TBA in the measuring malondi aldehyde activity. Biochemistry Analyt ica, 142(1984)290.

[5] Elstner E. F. Thin-cheromatography of lipid. Methods enzymology,Vol14;572.

[6] Information on http://link.springer.com/article/10.1007\%2FPL00000198

[7] Balld B, et al. Lipiid peroxidation and scavenging enzyme during exercise.J. Appl Physiol, 64(1988)1333

[8] Pu Junzong, Tang Pei. Research into the Influence of Different Amounts of Exercises on Animals' Immunity Indices . Chinese Journal of Sports Medicine.3(1996)170—173 
[9] Bi Qiuyun, Peng Li, Wang Qirong. Change in the Activities of CK and Other Enzymes that Perform the Same Biochemical Functions, LDH, ATL, AST in the Rats' Serum after Doing Increasing Load Exercises. Chinese Journal of Sports Medicine,5 (2006)12-18

[10]Wang Pei, Wang Guoqing. Influence of Overload Exercises on the Rats' Liver Function. Modern Rehabilitation,8,(2011)106-107

[11]Information on http://link.springer.com/article/10.2165\%2F00007256-200535030-00003

[12]Ye Jianfei, Yu Min, Ceng Haowang. Pathophysiology and Rehibitation of Overload Exercises. Building the Rats' Overload Exercise Model. Chinese Journal of Sports Medicine, 11(2014) $15-21$. 